\title{
Evaluation of Current Food Safety Practices at Various Food Establishments in Lahore
}

\author{
Muhammad Shehbaz \\ Faculty of Bio-Sciences, Department of Physiology, UVAS, Punjab, Pakistan
}

*Corresponding author: Muhammad Shehbaz, Faculty of Bio-Sciences, Department of Physiology, UVAS, Punjab, Pakistan, Tel: +9242-9921-1449-289; E-mail: umairmustafa_dt@yahoo.com

Received date: January 06, 2016; Accepted date: January 24, 2016; Published date: February 02, 2016

Copyright: ( 2016 Shehbaz M. This is an open-access article distributed under the terms of the Creative Commons Attribution License, which permits unrestricted use, distribution, and reproduction in any medium, provided the original author and source are credited.

\begin{abstract}
Food Safety is a scientific discipline describing handling, preparation and storage of foods in ways that prevent food borne illnesses. In present era there is much advancement in the process of product control and practices which are aimed to produce wholesome and safe food. Governments have imposed very strict quality and food safety assessment parameters for food establishments. All food establishments have not adopted practices to ensure food safety. In the present study the survey of 500 food establishments of Lahore were conducted to evaluate current food safety practices adopted by various food establishments. The evaluation was done by using structured questionnaire/checklist. The questionnaire/checklist detailed of food establishment, management and personnel, personal hygiene, establishment design and physical facilities, maintenance and sanitation of establishments, control of operations and consumer awareness with product information.
\end{abstract}

Keywords: Food safety; Hygiene; Food operators; Quality

\section{Review of Literature}

Barriers and benefits of Food Safety Management System (FSMS) implementation in food sector measured in survey study that was conducted in Harare province of Zimbabwe. A structured questionnaire as a tool was used for this survey. This study was comprises of fifty five questionnaires that were distributed among food establishments which only 30 received which were filled and completed. The survey showed that less financial resources and little investment by small companies is the main hurdles in implementing food safety. This survey also showed that implementation of Food Safety Management System (FSMS) also improve product quality which results in to the success of the company [1].

A survey was done to evaluate the level of hygiene in different food establishments supplying food direct to consumers in 2013 in three cosmopolitan cities of Europe. A total no of about 91 food businesses were included and surveyed in this study. An appropriate rating scheme was used for scoring the premises. Importance of food hygiene was justified by using another food safety dimension of consumer perception that is related to food safety and hygiene practices at these cities of Europe. In this survey 600 respondents were selected and interviewed. This study revealed that level of food safety and hygiene at various food establishments is best assessed on the basis of implementation of Hazard Analysis and Critical Control Point (HACCP) and all food establishments which have adopted and implemented HACCP they have higher level of food safety and hygienic practices. Extent of improvement of food safety knowledge and practices of employee through food safety training was examined in this study. Employee knowledge and practice for food safety was assessed and evaluated before and after the food safety trainings at their premises. A structured questionnaires prepared and was used to for evaluation of knowledge and practices of workers to check either they have adopted food safety practices or not. A restaurant performance of food safety program was also rated and assessed through a checklist. SPSS version 16 was used for statistical analysis of the data. As per methodology total 12 no of restaurants were included in this study which further divided into two groups one was named as intervention group. In this group all the participants were provided food safety training and second group was named as control group and no training was given to participants of this group. It was showed through this research that training have significant improvement in the knowledge and information of workers and food handlers their figure was 49.3 before the training which was raised up to 66.6 after the training. Sanitation and hygiene practices of employees and workers did no improve in this training. It was recommended through this research study that employee of the restaurants should provide job specific trainings with training material and this program should be continue to improve the food safety conditions at restaurants [2-4].

A survey on Food Safety Management system was also done in Siberia in 2012. Benefits, difficulties and costs for implementing Food Safety and Management System were studied in this survey. 77 food producers were analyzed in research. This research showed that $93.5 \%$ companies showed HACCP certification but only $6.5 \%$ had implemented HACCP, 9.1\% companies were showing ISO 22000 certification but only $1.3 \%$ had compliance. Finance in implementing food safety system was found as main hurdle. But to implement it is a key to improve food quality and safety which can increase output $[5,6]$.

A food safety survey was also conducted on Turkish poultry industry. This survey was comprises of 25 major meat producers that were compared. It was concluded that in implementation of ISO 22000 large firms adopted more strong plans as compared to small firms. Large firms have more financial support from government and as they are large producer so they have trained manpower to cope up with product contamination and produce healthier and safer products.

A research paper was published in UAE in which advantages and important factors which create awareness in food operators to implement the system were determined. A total 900 certified 
Page 2 of 8

companies were included in the survey among which $23.2 \%$ gave response. This survey showed that certified companies are more committed to their company status, improving their products continuously and more compliance to standards. It was also discussed that what the expected advantages of food safety system and what are the real benefits a company is achieving $[7,8]$.

257 retail food samples were tested in a study that was conducted in Novi Sad. In this study it was found $8.17 \%$ had contamination with Campylobacter, Salmonella and Listeria monocytogenes. The main sources of cross contamination in this sector are fresh meat cuts which they if not properly wash and save can cause severe food poisoning and are also main source of contamination. Campylobacter was found $8.8 \%$ samples of poultry and $10 \%$ samples of fresh meat. Salmonella prevalence was also in about $3.3 \%$ samples of poultry meat and Listeria monocytogenes found in 5\% samples of fresh meat and 3.3\% samples of other fresh meat $[9,10]$.

A research was conducted on food borne pathogens. Food borne pathogens are leading cause of disease and death in developing countries, killing an estimated 1.9 million people per year globally. It was estimated that up to one third of population were affected by microbiological food borne illness. The major factors were change in animal production systems and in the food chain. Both changes can cause corresponding changes in patterns of exposure to the pathogens. Five important food borne pathogens i.e. Salmonella spp, Campylobacter spp. E. coli, Toxoplasma gondiiand Cryptosporidium parvum were described [7]. Hazard Analysis Critical Control Point benefits and burdens in small companies were estimated for producing safe food. In this study burdens like record keeping, analyzing, training of employees, time and financial problems were discussed and certain benefits of HACCP were also revealed in research like legal protection, increasing trades and it creates a confidence in customers that product which they are using safe to eat. All factors for the compliance of food Safety Management System were highlighted in this research. The main purpose of this paper was to identify problems and their solution and developing strong system.

Hurdles and problems in the way of HACCP were discussed in 2001 in a research. These include all ways and procedures which becomes preventive in implementing HACCP. It may be producer's attitude, his commitment towards his customers, company size, available staff, less training of workers, no cooperation between company and certification bodies, no finance available, lack of record keeping practices, and small companies. It was concluded in the paper that successful implementation of HACCP is not possible until all these barriers be removed. Removal of these hurdles is necessary for producing safe food [11]. An assessment of Hazard Analysis Critical Control point procedures was done to check the food industry compliance with the system. This study focus on fulfilling the HACCP plan requirements. This paper depicted that food safety depends on the knowledge, talent and expertise of those people who are involved in implementation. It was reported that to control and monitor standards is necessary for meeting the food safety requirements. The purpose for assessing HACCP is to check the competency of food operators in which assessment of all processes, procedures and raw materials and finished products were done $[12,13]$.

In 1998 a survey report was presented on the implementation of Hazard Analysis Critical Control Point. Its main purpose was to getting the information about the hazards and shows the hurdles in the way of HACCP. The survey showed that $72.6 \%$ companies had implemented HACCP, 15\% were involved in registering their companies for HACCP and $12.6 \%$ did not use this system at all. The main problems in implementing HACCP were company size, financial status of company, number of employees, workers training and type of product [14].

\section{Statement of Problem}

Food is highly perishable commodity which can directly affect the health of a consumer. To ensure availability of safe and hygienic food to consumers is challenge for food service establishments and regulatory authorities. Although Punjab Food Authority has strict regulations and licensed about 10, 000 food establishments in Lahore in 2014. Still food establishments need to improve food safety practices to fulfill the gaps in implementation. Keeping in view the above situation the present study has been designed to evaluate the current food safety practices adopted by food establishments in Lahore.

\section{Method and Discussion}

The improvement of the concept of hygienic conditions of food was the outcome of spreads in accepting the co-relation of food, nutrition and health, which means an applied and innovative attitude to attain healthy life by possibly decreasing the risk of syndromes and diseases, and encouraging the healthy and fit status. Currently foodstuffs are not only to deliver essential nutrients to human body or just intentional to bear out appetite but also acting a part to evade the nutritional disorders and the physical and mental upgrading and satisfaction of the consumers.

Safe food diets show an exceptional job in this regard. The production and promotion of these categories need extra ordinary care exertions including the determination of hygienic conditions, food handling and evaluating their food safety, the composition and ingredients of foodstuff, may get effected during developing and food processing and food storage resulting in the fluctuation of bioavailability [15]. Hence, awareness of the customer is required and in order to secure authorization for health improving claim efficacy study is mandatory. An important thing is the food safety within food sector. Because of limited consumer of the developed countries knows about food safety and lack of knowledge of their effects on health particularly in third world countries like Pakistan this research was planned to report at least some part such poor understanding whereas the outcomes and the recommendations has been discussed below.

The present study was conducted to evaluate the current food safety practices at various food establishments e.g. fast food chains, dairy and milk shops, sweets and bakers, bottled water and beverages, marriage halls and canteens, restaurants and hotels, cafes and canteens and manufacturing industries from all towns of district Lahore under jurisdiction of Punjab Food Authority. Data was collected from different towns of Lahore as $6 \%$ fast food chains, 28.1 dairy and milk shops, 15.8 sweets and bakers, $6.8 \%$ bottled water and beverages, $6 \%$ marriage halls and caterings, $23.2 \%$ restaurants and hotels, $10.8 \%$ cafes and canteens and 3.2\% manufacturing industries.

The population in the study was selected from 8 different types of food categories which comprised of 30 (6\%) from fast food chains, 141 (28.1\%) from dairy and milk shops, $79(15.8 \%)$ from sweets and bakers, $34(6.8 \%)$ from bottled water and beverages, 30 (6\%) from marriage halls and caterings, 116 (23.8\%) from restaurants and hotels, $54(10.8 \%)$ from cafes and canteens and $16(3.2 \%)$ from manufacturing industries. 
The population was selected from each town to District Lahore which was comprised of 69 (13.8\%) were from Allama Iqbal Town, 43 (8.6\%) from Aziz Bhatti Town, 67 (13.4\%) from Data Ganj Buksh Town, 78 (15.6\%) from Gulberg Town, 37 (7.4\%) from Nishter Town, 66 (13.2\%) from Ravi Town, 72 (14.4\%) from Samna bad Town, 53 (10.6\%) from Shalimar Town and $15(3 \%)$ from Wahga Town.

The selected population in the study was categorized according to their number of years of establishment status. Out of the total studied population (100\%), only $1.2 \%$ was 1 year old, $7.2 \%$ were 2 years old, $38.5 \%$ were 3 years old and 52.9 were greater than 3 years in the above spectrum.

\section{Management and personnel}

According to the appointment of full time competent person incharge (PIC) at food establishment, Out of 500 food establishment only $17 \%$ has appointed and $83 \%$ have not appointed the full time competent person in charge on their premises. The $p$ value is 0.000 which showed significant results. According to knowledge and information of person incharge (PIC) at food establishment, Out of 500 food establishments only $14.8 \%$ have proper knowledge and information and $85.2 \%$ have not proper knowledge and information. The $\mathrm{p}$ value is 0.000 which showed significant results.

According to passing of written examination which is acceptable to food authority for person incharge (PIC) at food establishment, Out of 500 foods establishment only $1.8 \%$ have passed written exam and 98.2\% have not passes any type of exam which is acceptable to food authority. The $\mathrm{p}$ value is 0.000 which showed significant results. The significance shows that the food establishments were differ from each other according to PIC.

In the appearance of name of PIC behind the cash counter and any other place which is clearly visible to customers. Out of 500 foods establishment only $2 \%$ have mentioned and $98 \%$ have not mentioned name behind the cash counter. The $\mathrm{p}$ value is 0.000 which showed significant results. The significant values show the variation in the food outlets. In the sense of effective supervision by PIC in implementing safe food practices, conduct regular inspections of the food premises, address potential food risks and take appropriate actions. Out of 500 foods establishment only on $17.6 \%$ food establishments PIC have effective supervision and on $82.4 \%$ food establishments there is no proper supervision by PIC. The $\mathrm{p}$ value is 0.000 which showed significant results tells that the food outlets required more attention.

In the responsibilities of PIC is the major duty to conduct the awareness sessions for the food handlers. For relevant trainings of staff, employees and contractors before commencing to work, Out of 500 foods establishment $24 \%$ require urgent improvement, $17 \%$ require major improvement, on $50 \%$ food establishments improvement necessary, $6 \%$ are generally satisfactory, only $1 \%$ are good and $2 \%$ are in very good rating. The $\mathrm{p}$ value is 0.000 which showed significant results. The results of $\mathrm{p}$ value shows that the food outlets require more training sessions of awareness.

In the sense of placement of documented program of relevant food safety trainings in food establishments, Out of 500 foods establishment $24 \%$ require urgent improvement, $17 \%$ require major improvement, on $44 \%$ food establishments improvement necessary, 6\% are generally satisfactory, only $2 \%$ are good and $1 \%$ are in very good rating. The $p$ value is 0.000 which showed significant results. The highly significance results tells the much variation in different outlets of foods. According to other countries food safety the documentation is very compulsory especially in New Zealand. The New Zealands food safety documentation survey is much differ because the New Zealands food authority performance is better than Pakistan.

According to management and personnel condition of different food outlets, Out of 500 foods establishment $36 \%$ require urgent improvement, $44 \%$ require major improvement, on $12 \%$ food establishments improvement necessary, $4 \%$ are generally satisfactory, only $2 \%$ are good and $2 \%$ are in very good rating. The p value is 0.000 which showed significant results. The food outlets are present in Pakistan have different levels. Every food outlet according to their level knows about management and personnel conditions.

The health of food handlers plays the pivotal role in the health of consumers. Good and healthy employees are the backbone of food factory. According to employee good health and good eye sight, Out of 500 foods establishment $24 \%$ require urgent improvement, $17 \%$ require major improvement, on $40 \%$ food establishments improvement necessary, $26 \%$ are generally satisfactory, only $5 \%$ are good and $0 \%$ are in very good rating. The $\mathrm{p}$ value is 0.000 which showed significant results. The restaurants have the much difference due to their levels best. In other countries good health of employee is very important especially in England.

The medical fitness is very important for food handlers. According to employee annual medical screening and fitness tests, Out of 500 foods establishment $24 \%$ require urgent improvement, $17 \%$ require major improvement, on $26 \%$ food establishments improvement necessary, $37 \%$ are generally satisfactory, only $3 \%$ are good and $1 \%$ are in very good rating. The $\mathrm{p}$ value is 0.000 which showed significant results. The significant data shows the more improvement is require in Pakistan according to employees health.

Illness and especially communicable diseases of employees is the major risk in safe food preparation. According to symptoms of illnesses and communicable diseases, Out of 500 foods establishment $24 \%$ require urgent improvement, $17 \%$ require major improvement, on $33 \%$ food establishments improvement necessary, $30 \%$ are generally satisfactory, only $3 \%$ are good and $0 \%$ are in very good rating. The $p$ value is 0.000 which showed significant results. The significant data tells the situation of different food outlets in the Pakistan [16-18].

Wounds and exposed parts of body is a major risk of cross contamination. Out of 500 foods establishment $24 \%$ require urgent improvement, $17 \%$ require major improvement, on $21 \%$ food establishments improvement necessary, $42 \%$ are generally satisfactory, only $4 \%$ are good and $0 \%$ are in very good rating. The p value is 0.000 which showed significant results. The significant results showed the much variation in different food establishments.

If any food handler suffer from in any disease it is compulsory to report in the office of food establishment. According to instructions to report diseases, Out of 500 foods establishment $24 \%$ require urgent improvement, $17 \%$ require major improvement, on $33 \%$ food establishments improvement necessary, $30 \%$ are generally satisfactory, only $3 \%$ are good and $0 \%$ are in very good rating. The $p$ value is 0.000 which showed significant results. The results of food handlers, disease reporting tells the major variation according to different food establishments. It is due to the presence of different levels of food establishments in Pakistan.

The food suppliers provide the supply of different types of raw food in different food establishments. A supplier health is also plays the pivotal role for the communication of diseases. According to 
implementation of health questionnaire for visitors and contractors, Out of 500 foods establishment 24\% require urgent improvement, $17 \%$ require major improvement, on $33 \%$ food establishment's improvement necessary, $30 \%$ are generally satisfactory, only $3 \%$ are good and $0 \%$ are in very good rating. The $\mathrm{p}$ value is 0.000 which showed significant results. The variation in standards of food establishment is present in Pakistan because a lot of levels of food establishments are present in Pakistan.

\section{Personnel hygiene practices}

Jewelry and watches wearing during the processing of foods may be the major physical contamination. So it is restricted during processing of foods according to HACCP and WHO. But in the situation of different food establishments in Pakistan, Out of 500 foods establishment $24 \%$ require urgent improvement, $17 \%$ require major improvement, on $37 \%$ food establishments improvement necessary, $20 \%$ are generally satisfactory, only $4 \%$ are good and $2 \%$ are in very good rating. The $\mathrm{p}$ value is 0.000 which showed significant results. According to wearing of wearing of jewelry, Out of 500 foods establishment $24 \%$ require urgent improvement, $17 \%$ require major improvement, on $32 \%$ food establishments improvement necessary, $25 \%$ are generally satisfactory, only $4 \%$ are good and $2 \%$ are in very good rating. The $p$ value is 0.000 which showed significant results. The variation between different food establishments is due to standards differentiation.

The presence of volatile compounds in food processing area is the major odor contamination source. According to use of excessive perfumes while working in food preparation areas.. Out of 500 foods establishment $24 \%$ require urgent improvement, $17 \%$ require major improvement, on $32 \%$ food establishments improvement necessary, $25 \%$ are generally satisfactory, only $4 \%$ are good and $2 \%$ are in very good rating. The $\mathrm{p}$ value is 0.000 which showed significant results. According to results in Pakistan some food establishments need urgent improvement in this discipline.

Major food borne illness is due to poor personnel hygienic condition of food handler. Hand washing and wearing of gloves protects from food borne illness. According to employee hand washing practices, Out of 500 foods establishment $24 \%$ require urgent improvement, $17 \%$ require major improvement, on $36 \%$ food establishments improvement necessary, $19 \%$ are generally satisfactory, only $4 \%$ are good and $2 \%$ are in very good rating. The $p$ value is 0.000 which showed significant results. In some food establishments urgent improvement is require in staff food safety education. Personal habits of food handlers are also a seeking point either employee has good or bad personal habits like spitting. According to smoking, spitting and using tobacco inside food preparation area, Out of 500 foods establishment $24 \%$ require urgent improvement, $17 \%$ require major improvement, on $20 \%$ food establishments improvement necessary, $6 \%$ are generally satisfactory, only $9 \%$ are good and $0 \%$ are in very good rating. The $\mathrm{p}$ value is 0.000 which showed significant results. According to chewing, sneezing and coughing over unprotected food, Out of 500 foods establishment $24 \%$ require urgent improvement, $17 \%$ require major improvement, on $20 \%$ food establishments improvement necessary, $19 \%$ are generally satisfactory, only $5 \%$ are good and $0 \%$ are in very good rating. The $\mathrm{p}$ value is 0.000 which showed significant results. Urgent improvement is necessary in this discipline for safe food production.

Gloves wearing are very compulsory for safe food production. According to touching ready to eat foods with bare hands, Out of 500 foods establishment $24 \%$ require urgent improvement, $17 \%$ require major improvement, on $36 \%$ food establishments improvement necessary, $20 \%$ are generally satisfactory, only $9 \%$ are good and $0 \%$ are in very good rating. The $\mathrm{p}$ value is 0.000 which showed significant results. The significant results showed the much value addition require for safe food production.

Food tasting SOPs developed in developed countries. According to tasting food with fingers and touching hair, nose, eyes and ears, Out of 500 foods establishment $24 \%$ require urgent improvement, $17 \%$ require major improvement, on $18 \%$ food establishments improvement necessary, $17 \%$ are generally satisfactory, only $5 \%$ are good and $2 \%$ are in very good rating. The $\mathrm{p}$ value is 0.000 which showed significant results. In Pakistan much improvement needs.

\section{Food establishment infrastructure}

Food establishments have the specific infrastructure and Governments issues the permission letter to establish a food outlet. According to prior approval of food establishment layout, Out of 500 foods establishment only $12 \%$ met the criteria and $88 \%$ do not met the criteria. In fast food $23 \%$ and $77 \%$ do not follow, in dairy and milk shops, no any establishment follow the criteria, sweets and bakers follow the criteria and $92 \%$ do not follow the criteria, bottled water and beverages $6 \%$ follow and $94 \%$ do not follow, in marriage halls and caterings 3\% follow and $97 \%$ do not follow the criteria, restaurants and hotels $22 \%$ follow the criteria and $78 \%$ do not follow, cafes and canteens 11 follow and $89 \%$ do not follow and in manufacturing industry $63 \%$ follow the criteria and $38 \%$ do not follow the criteria. The $\mathrm{p}$ value is 0.000 which showed significant results. In Pakistan there is no proper layout for foods establishments so much improvement is necessary for proper layouts of food establishments. In proper layout safety sights are measured and layouts of food establishments are designed. According to site safety from potential sources of contamination, Out of 500 foods establishment only $43 \%$ met the criteria and $57 \%$ do not met the criteria. Urgent improvement is necessary for corrective actions and from the prevention of cross contamination.

Floor of food processing area must be cleaned, durable and nonslippery. According to floors cleanliness and durability, Out of 500 foods establishment only $25 \%$ met the criteria and $75 \%$ do not met the criteria. Urgent improvement is necessary in proper layouts of food processing area. According to ceilings conditions and signs of pest infestation, Out of 500 foods establishment only $36 \%$ met the criteria and $64 \%$ do not met the criteria.

In food establishments toilets must be separated from food processing area. Toilets are the major risk of food borne illness if the toilets are present near food processing area. According to availability of toilets which are segregated from food production and preparation areas, Out of 500 foods establishment only $40 \%$ met the criteria and $60 \%$ do not met the criteria. 60\% layouts are not proper in Pakistan; however urgent improvement is necessary from the prevention of food borne illness. According to drains effectiveness to remove waste water. Out of 500 foods establishment only $29 \%$ met the criteria and $71 \%$ do not met the criteria. Stairways, mezzanines and lobbies are always located away from food processing area. According to stairways and mezzanines location, Out of 500 foods establishment only $40 \%$ met the criteria and $60 \%$ do not met the criteria. And according to waste and debris containers location, Out of 500 foods establishment only $29 \%$ met the criteria and $71 \%$ do not met the criteria. Urgent improvement is necessary for proper food safety. 
From the prevention of cross contamination it is compulsory that the working areas and surfaces neat and clean. According to condition of working surfaces, Out of 500 foods establishment only $32 \%$ met the criteria and $68 \%$ do not met the criteria. $68 \%$ food establishments need urgent improvement for the production of safe food. Lights and bulbs and proper illuminations are compulsory for handling the safe food production. According to condition shield coated and shatter resistant light bulbs, Out of 500 foods establishment only $28 \%$ met the criteria and $72 \%$ do not met the criteria. Improvements need in this discipline. According to heating, ventilation and air conditioning, Out of 500 foods establishment only $44 \%$ met the criteria and $56 \%$ do not met the criteria. According to designated changing facilities for personnel, staff and visitors, Out of 500 foods establishment only $43 \%$ met the criteria and $57 \%$ do not met the criteria. According to availability of sufficient quantity of water at appropriate temperature, Out of 500 foods establishment only $25 \%$ met the criteria and $75 \%$ do not met the criteria. Hand soaps and sanitizers have the germicidal effect and compulsory in food processing areas to germs free hands. According to availability of liquid soap and hand sanitizers, Out of 500 foods establishment only $28 \%$ met the criteria and $72 \%$ do not met the criteria. Urgent improvements are necessary for the production of safe foods.

According to availability of water taps and hands free operation in high risk areas, Out of 500 foods establishment only $29 \%$ met the criteria and $71 \%$ do not met the criteria. According to availability of Toilets which are segregated from Food Production and Preparation Areas, Out of 500 foods establishment only $40 \%$ met the criteria and $60 \%$ do not met the criteria. According to availability of basin, soap and water in staff toilets, Out of 500 foods establishment only $32 \%$ met the criteria and $68 \%$ do not met the criteria. According to visibility of advisory signs at hand washing areas, Out of 500 foods establishment only $44 \%$ met the criteria and $56 \%$ do not met the criteria.

\section{Food Establishment Maintenance}

Cleaning and disinfection of foods processing area is very compulsory for safe food production. According to cleaning and disinfection area program, Out of 500 foods establishment $40 \%$ require urgent improvement, 20\% require major improvement, on 19\% food establishments improvement necessary $19 \%$ are generally satisfactory, only $1 \%$ are good and $2 \%$ are in very good rating. The $p$ value is 0.000 which showed significant results. According to availability of sufficient and suitable cleaning material, Out of 500 foods establishment $40 \%$ require urgent improvement, $16 \%$ require major improvement, on $23 \%$ food establishments improvement necessary $18 \%$ are generally satisfactory, only $2 \%$ are good and $2 \%$ are in very good rating. The $p$ value is 0.000 which showed significant results. According to floor surfaces, Out of 500 foods establishment $40 \%$ require urgent improvement, $20 \%$ require major improvement, on $19 \%$ food establishments improvement necessary $18 \%$ are generally satisfactory, only $1 \%$ are good and $2 \%$ are in very good rating. The $p$ value is 0.000 which showed significant results. It is compulsory for the prevention of food processing area from biofilms; to prevent from biofilms the floor must be free from Cracks, Holes, Gaps and Flaking Material to Maintain Good Conditions.

Biofilms in food processing area is the major risk for unsafe food. According to junctions between walls and ceilings, Out of 500 foods establishment $40 \%$ require urgent improvement, $16 \%$ require major improvement, on $21 \%$ food establishments improvement necessary $20 \%$ are generally satisfactory, only $2 \%$ are good and $2 \%$ are in very good rating. The $p$ value is 0.000 which showed significant results. The food outlets have the much variation due to different standards of food establishments. According to food contact surfaces of equipment's and utensils, Out of 500 foods establishment $40 \%$ require urgent improvement, $16 \%$ require major improvement, on $21 \%$ food establishments improvement necessary $20 \%$ are generally satisfactory, only $2 \%$ are good and $2 \%$ are in very good rating. The $p$ value is 0.000 which showed significant results.

Pot washing and cleaning of equipment's area must be separate from food processing area. According to separate placement of cleaned and disinfected equipment's and utensils, Out of 500 foods establishment $40 \%$ require urgent improvement, $16 \%$ require major improvement, on $21 \%$ food establishments improvement necessary $20 \%$ are generally satisfactory, only $2 \%$ are good and $2 \%$ are in very good rating. The significance value is 0.000 which showed significant results. Food grade chemicals and detergents must be available in food processing area. According to availability of approved and food grade chemicals and detergents. Out of 500 foods establishment $40 \%$ require urgent improvement, $16 \%$ require major improvement, on $21 \%$ food establishments improvement necessary $20 \%$ are generally satisfactory, only $2 \%$ are good and $2 \%$ are in very good rating. The $p$ value is 0.000 which showed significant results. Urgent improvements required in some food establishments in Pakistan.

Fumigation contribution has the pivotal role from the prevention of pests and rodents. According to emplacement of preventive pest control program, Out of 500 foods establishment 39\% require urgent improvement, $23 \%$ require major improvement, on $19 \%$ food establishments improvement necessary $16 \%$ are generally satisfactory, only $0 \%$ are good and $3 \%$ are in very good rating. The $p$ value is 0.000 which showed significant results. According to location and placement of bait stations, Out of 500 foods establishment 39\% require urgent improvement, $22 \%$ require major improvement, on $20 \%$ food establishments improvement necessary $16 \%$ are generally satisfactory, only $0 \%$ are good and $3 \%$ are in very good rating. The $p$ value is 0.000 which showed significant results. According to location and operational status of electric fly killers. Out of 500 foods establishment $39 \%$ require urgent improvement, $23 \%$ require major improvement, on $19 \%$ food establishments improvement necessary $16 \%$ are generally satisfactory, only $0 \%$ are good and $3 \%$ are in very good rating. The $p$ value is 0.000 which showed significant results. According to corrective actions on pest infestation, Out of 500 foods establishment $39 \%$ require urgent improvement, $22 \%$ require major improvement, on $18 \%$ food establishments improvement necessary $18 \%$ are generally satisfactory, only $0 \%$ are good and $3 \%$ are in very good rating. The $p$ value is 0.000 which showed significant results. According to availability of records of pest control inspections, recommendations and corrective actions taken, Out of 500 foods establishment 39\% require urgent improvement, $22 \%$ require major improvement, on $18 \%$ food establishments improvement necessary $18 \%$ are generally satisfactory, only $0 \%$ are good and $3 \%$ are in very good rating. The $p$ value is 0.000 which showed significant results.

According to pest control trend analysis, Out of 500 foods establishment 39\% require urgent improvement, 22\% require major improvement, on $18 \%$ food establishments improvement necessary $18 \%$ are generally satisfactory, only $0 \%$ are good and $3 \%$ are in very good rating. The $\mathrm{p}$ value is 0.000 which showed significant results. According to records of pesticide usage, Out of 500 foods establishment $39 \%$ require urgent improvement, $22 \%$ require major improvement, on $20 \%$ food establishments improvement 
necessary $16 \%$ are generally satisfactory, only $0 \%$ are good and $3 \%$ are in very good rating. The $\mathrm{p}$ value is 0.000 which showed significant results.

According to records of pesticide usage, Out of 500 foods establishment $39 \%$ require urgent improvement, $22 \%$ require major improvement, on $20 \%$ food establishments improvement necessary $16 \%$ are generally satisfactory, only $0 \%$ are good and $3 \%$ are in very good rating. The $\mathrm{p}$ value is 0.000 which showed significant results. It is compulsory that the every food establishment sure the HACCP system in food processing area. Fumigate the area of food processing properly and scheduled and prepare the documents. In Pakistan today the condition of pest control is not good and urgent improvement require in this regard. Waste management is a big issue that how, when and in that container waste the garbage of food. According to location of solid waste containers, Out of 500 foods establishment 35\% require urgent improvement, $12 \%$ require major improvement, on $35 \%$ food establishments improvement necessary $16 \%$ are generally satisfactory, only $2 \%$ are good and $0 \%$ are in very good rating. The $\mathrm{p}$ value is 0.000 which showed significant results. In standards dry and wet garbage rooms separately available in food establishment and waste the garbage in polythene bags. According to cleaning frequency of garbage, swage and solid waste containers.. Out of 500 foods establishment $25 \%$ require urgent improvement, $12 \%$ require major improvement, on $33 \%$ food establishments improvement necessary $18 \%$ are generally satisfactory, only $2 \%$ are good and $0 \%$ are in very good rating. The $p$ value is 0.000 which showed significant results.

According to sufficient and accessible solid waste containers, Out of 500 foods establishment $35 \%$ require urgent improvement, $12 \%$ require major improvement, on $34 \%$ food establishments improvement necessary $18 \%$ are generally satisfactory, only $1 \%$ are good and $0 \%$ are in very good rating. The $\mathrm{p}$ value is 0.000 which showed significant results. According to approval of solid waste collector from environment protection agency, Out of 500 foods establishment only $1 \%$ follow the criteria and $99 \%$ do not follow the criteria. The $\mathrm{p}$ value is 0.000 which showed significant results.

\section{Raw material inspection}

The quality of foods directly related to raw material. If the quality of raw material excellent the food quality is also good. According to approved sources for food and food ingredients. Out of 500 foods establishment only $12 \%$ follow the criteria and $99 \%$ do not follow the criteria. The p value is 0.000 which showed significant results. Different establishments use the different types of raw material. Some establishments prepare their own standards and have good quality of processed food. According to checking and inspection of raw materials at receiving, Out of 500 foods establishment only $28 \%$ follow the criteria and $72 \%$ do not follow the criteria. The $p$ value is 0.000 which showed significant results. According to packaging and labeling as per Punjab Food Authority Act, 2011, Out of 500 foods establishment only $36 \%$ follow the criteria and $64 \%$ do not follow the criteria. The $p$ value is 0.000 which showed significant results. According to retention of invoices and receipts, Out of 500 foods establishment only $29 \%$ follow the criteria and $71 \%$ do not follow the criteria. The $\mathrm{p}$ value is 0.000 which showed significant results. According to food serving temperature, Out of 500 foods establishment only $83 \%$ follow the criteria and $17 \%$ do not follow the criteria. The $p$ value is 0.000 which showed significant results. According to availability of hot holding devices, Out of 500 foods establishment only $25 \%$ follow the criteria and $75 \%$ do not follow the criteria. The $\mathrm{p}$ value is 0.000 which showed significant results.

According to checking of cooking temperature with calibrated thermometer, Out of 500 foods establishment only 23\% follow the criteria and $77 \%$ do not follow the criteria. The $\mathrm{p}$ value is 0.000 which showed significant results. According to food products storage and handling. Out of 500 foods establishment only $28 \%$ follow the criteria and $72 \%$ do not follow the criteria. The p value is 0.000 which showed significant results. According to availability of time and temperature measuring devices, Out of 500 foods establishment only $28 \%$ follow the criteria and $72 \%$ do not follow the criteria. The $p$ value is 0.000 which showed significant results. According to availability of dry food storage, Out of 500 foods establishment only $28 \%$ follow the criteria and $72 \%$ do not follow the criteria. The $p$ value is 0.000 which showed significant results. Dry food stores is very compulsory from the prevention of food cross contamination. Food transportation temperature maintenance is compulsory for the prevention of food from spoilage. According to food transportation and distribution temperature, Out of 500 foods establishment only 32\% follow the criteria and $56 \%$ do not follow the criteria. The $p$ value is 0.000 which showed significant results. According to separate placement of cleaned and disinfected equipment's and utensils, Out of 500 foods establishment $42 \%$ require urgent improvement, $7 \%$ require major improvement, on $31 \%$ food establishments improvement necessary $11 \%$ are generally satisfactory, only $8 \%$ are good and $0 \%$ are in very good rating. The $\mathrm{p}$ value is 0.000 which showed significant results. According to proper washing of raw fruits and vegetables, Out of 500 foods establishment $42 \%$ require urgent improvement, $7 \%$ require major improvement, on $26 \%$ food establishments improvement necessary $17 \%$ are generally satisfactory, only $8 \%$ are good and $0 \%$ are in very good rating. The $\mathrm{p}$ value is 0.000 which showed significant results. According to prohibition of food in lockers, dressing rooms and garbage rooms, Out of 500 foods establishment $42 \%$ require urgent improvement, $7 \%$ require major improvement, on $14 \%$ food establishments improvement necessary $32 \%$ are generally satisfactory, only $5 \%$ are good and $0 \%$ are in very good rating. The $p$ value is 0.000 which showed significant results. Lockers are area where employees change their uniforms and taking bath. So the food eating is prohibited in those areas.

Different types of ice making machines are used in food processing establishments. According to usage of dirty ice, Out of 500 foods establishment $42 \%$ require urgent improvement, $7 \%$ require major improvement, on $26 \%$ food establishments improvement necessary $17 \%$ are generally satisfactory, only $8 \%$ are good and $0 \%$ are in very good rating. The $\mathrm{p}$ value is 0.000 which showed significant results. The significant results showed about the different restaurants and food establishments which use the different types of ice and ice is a major source of cross contamination.

\section{Prevention of physical and chemical contamination}

According to installation of detection and rejection devices of devices, Out of 500 foods establishment only $11 \%$ follow the criteria and $89 \%$ do not follow the criteria. The p value is 0.000 which showed significant result. According to usage of adequate covers on equipment and containers, Out of 500 foods establishment $37 \%$ require urgent improvement, $20 \%$ require major improvement, on $22 \%$ food establishments improvement necessary $17 \%$ are generally satisfactory, only $4 \%$ are good and $0 \%$ are in very good rating. The $\mathrm{p}$ value is 0.000 which showed significant results. According to product contamination 
Page 7 of 8

with glass, wood, brittle and ceramics, Out of 500 foods establishment $37 \%$ require urgent improvement, $11 \%$ require major improvement, on $29 \%$ food establishments improvement necessary $14 \%$ are generally satisfactory, only $4 \%$ are good and $0 \%$ are in very good rating. The $p$ value is 0.000 which showed significant results. Different food establishments differ from each other according to their standards. According to use of food grade chemicals, Out of 500 foods establishment $37 \%$ require urgent improvement, $11 \%$ require major improvement, on $29 \%$ food establishments improvement necessary $14 \%$ are generally satisfactory, only $8 \%$ are good and $0 \%$ are in very good rating. The $\mathrm{p}$ value is 0.000 which showed significant results.

\section{Management of food allergens}

The new researches in the world explore the food allergy and prevent the peoples from allergen foods. According to declaration of most common food allergenic ingredients, Out of 500 foods establishment only $02 \%$ follow the criteria and $98 \%$ do not follow the criteria. The significance value is 0.000 which showed significant results. According to presence of allergenic ingredients on labels, Out of 500 foods establishment only $02 \%$ follow the criteria and $98 \%$ do not follow the criteria. The $p$ value is 0.000 which showed significant results. According to availability of full time competent person to deal with allergenic foods and communicate to customers. Out of 500 foods establishment only $02 \%$ follow the criteria and $98 \%$ do not follow the criteria. The significance value is 0.000 which showed significant results. The significant results showed the food allergens condition in Pakistan. Urgent improvement required in Pakistan to meet the international standards. In other countries like USA the food industries and food outlets have the education about allergens, but in Pakistan consumers are unaware about food allergens.

\section{Food product traceability and recalls}

In food processing industries, it is compulsory to fix the batch code, date and product number and time. According to registration and licensing with Punjab Food Authority, Out of 500 foods establishment only $100 \%$ follow the criteria and $0 \%$ do not follow the criteria. The $\mathrm{p}$ value is 0.000 which showed significant results. The results are highly significant. There is no more improvement required in this discipline and food establishments have good work. According to identification and traceability of lots, Out of 500 foods establishment only $37 \%$ follow the criteria and $63 \%$ do not follow the criteria. The significance value is 0.000 which showed significant results.

According to labeling and identification to facilitate traceability, Out of 500 foods establishment only $37 \%$ follow the criteria and $63 \%$ do not follow the criteria. The significance value is 0.000 which showed significant results. According to withdrawals and recalls of food products, Out of 500 foods establishment only $00 \%$ follow the criteria and $100 \%$ do not follow the criteria. The $\mathrm{p}$ value is 0.000 which showed significant results. According to availability of customer complaints records, Out of 500 foods establishment only $37 \%$ follow the criteria and $63 \%$ do not follow the criteria. The $p$ value is 0.000 which showed significant results. According to investigation and corrective actions on food safety customer complaints, Out of 500 foods establishment only $37 \%$ follow the criteria and $63 \%$ do not follow the criteria. The $\mathrm{p}$ value is 0.000 which showed significant results. According to availability of handling information's on food products, Out of 500 foods establishment only $31 \%$ follow the criteria and $69 \%$ do not follow the criteria. The significance value is 0.000 which showed significant results. It is compulsory for the food establishments to satisfy their consumers. Food establishments maintain their reputation and improve the goodness.

\section{Availability of Nutritional Information's for Awareness of Consumers about Their Health}

According to availability of nutritional information for awareness of consumers about their health, Out of 500 foods establishment only $14 \%$ follow the criteria and $86 \%$ do not follow the criteria. The p value is 0.000 which showed significant results. The current food safety situation in Pakistan is a pressing challenge and will continue to have serious implications to the nation's economic infrastructure if the corrective measures are not taken in a timely manner. Increases in the prevalence of foodborne illnesses especially diarrhea among children, is alarming, as Pakistan's future depends upon a healthier nation. Moreover, there is a dire need to scientifically tackle the food safety issue in Pakistan. Evidence from the literature suggests that majority of Pakistani foods are drastically implicated with contaminants. Food hygiene training as a means of improving food safety, strong consumer protection groups, awareness of food safety, and the right to acquire safe food, strict adherence to food laws, new legislation with changing food safety perspectives, and the recognition of this challenging problem by all stakeholders are some of the practical, systematic, and aggressive steps that can be taken to curtail the risk of microbiological and chemical foodborne diseases and to minimize the health consequences associated with the consumption of unsafe foods in Pakistan.

\section{Summary}

Food Safety is a scientific discipline describing handling, preparation and storage of foods in ways that prevent food borne illnesses. In present era there is much advancement in the process of product control and practices which are aimed to produce wholesome and safe food. Governments have imposed very strict quality and food safety assessment parameters for food establishments. In the present study the survey of 500 food establishments of Lahore were conducted to evaluate current food safety practices adopted by various food establishments. The evaluation was done by using structured questionnaire/checklist. The questionnaire/checklist detailed of food establishment, management and personnel, personal hygiene, establishment design and physical facilities, maintenance and sanitation of establishments, control of operations and consumer awareness with product information. SPSS version 16 was used for all data analyses. Descriptive statistics including frequencies, means, and standard deviations were calculated for all variables as appropriate. The project highlighted the situation of current food safety practices and gaps in implementation adopted by various food establishments in Lahore. Evidence from the literature suggests that majority of Pakistani foods are drastically implicated with contaminants. Food hygiene training as a means of improving food safety, strong consumer protection groups, awareness of food safety, and the right to acquire safe food, strict adherence to food laws, new legislation with changing food safety perspectives, and the recognition of this challenging problem by all stakeholders are some of the practical, systematic, and aggressive steps that can be taken to curtail the risk of microbiological and chemical foodborne diseases and to minimize the health consequences associated with the consumption of unsafe foods in Pakistan. 
Citation: Shehbaz M (2016) Evaluation of Current Food Safety Practices at Various Food Establishments in Lahore. J Food Process Technol 7: 559. doi:10.4172/2157-7110.1000559

Page 8 of 8

\section{References}

1. Macheka L, Manditsera FA, Ngadze RT, Mubaiwa J, Nyanga LK, et al (2013) Barriers, benefits and motivation factors for the implementation of food safety management system in the food sector in Harare Province, Zimbabwe. Food Control 34: 126-131.

2. Tsola E, Drosinos E, Zoiopoulos P (2008) Impact of poultry slaughter housep modernisation and updating of food safety management systems on the microbiological quality and safety of products. Food Control 19: 423-431.

3. Afzal M (2010) Re-designing smallholder dairy production in Pakistan. Pak Vet J 30:187-190.

4. Tahir S, Anwar T, Ahmad I, Aziz S, Mohammad A, et al. (2001) Determination of pesticide residues in fruits and vegetables in Islamabad market. J Environ Biol 22: 71-74.

5. Crutchfield SR, Buzby JC, Roberts T, Ollinger M, Lin C-TJ, et al. (1997) Economic Assessment of Food Safety Regulations: The New Approach to Meat and Poultry Inspection. United States Department of Agriculture, Economic Research Service. Food Policy 25: 223-228.

6. Holleran E, Bredahl ME, Zaibet L (1999) Private incentives for adopting food safety and quality assurance. Food policy 24: 669-683.

7. Hussain I, Anwar J, Munawar MA, Asi M R (2008) Variation of levels of aflatoxinM1 in raw milk from different localities in the central areas of Punjab, Pakistan. Food Control 19: 1126-1129.

8. Hussain I, Shahid Mahmood M, Akhtar M, Khan A (2007) Prevalence of Campylobacter species in meat, milk and other food commodities in Pakistan. Food Microbiol 24: 219-222.

9. Iqbal SZ, Paterson RR, Bhatti IA, Asi MR, Sheikh MA, et al. (2010) Aflatoxin B1 in chilies from the Punjab region, Pakistan. Mycotoxin Res 26: 205-209.
10. Jaffar M, Shah MH, Shaheen N, Khaliq A, Tariq SR, et al. (2004) Pre and post-expiry metal levels in canned dry milk. Nut Food Sci J 34: 65-71.

11. Panisello PJ, Quantick PC (2001) Technical barriers to hazard analysis critical control point (HACCP). Food Control 12: 165-173.

12. Jacxsens L, Kussaga J, Luning P, Van der Spiegel M, Devlieghere F, et al. (2009) A microbial assessment scheme to measure microbial performance of food safety management systems. International Journal of Food Microbiology 134: 113-125.

13. Jacxsens L, Luning P, Marcelis W, van Boekel T, Rovira J, et al. (2011) Tools for the performance assessment and improvement of food safety management systems. Trends in Food Science \& Technology 22: 80-89.

14. Panisello PJ, Quantick PC, Knowles MJ (1999) Towards the implementation of HACCP: results of a UK regional survey. Food Control 10: 87-98.

15. Akhtar S, Naz S, Sultan MT, Nasir M, Ahmad A, et al. (2010) Physico chemical attributes and heavy metal content of mangoes (mangifera indica L) cultivated in different regions of Pakistan. Pak J Bot 42: 2691-2702.

16. Jenson I, Sumner J (2012) Performance standards and meat safetydevelopments and direction. Meat Sci 92: 260-266.

17. Al-Kandari D, Jukes DJ (2011) Incorporating HACCP into national food control systems-Analyzing progress in the United Arab Emirates. Food Control 22: 851-861.

18. Smith J, Toupin C, Gagnon B, Voyer R, Fiset P, et al. (1990) A hazard analysis critical control point approach (HACCP) to ensure the microbiological safety of sous vide processed meat/pasta product. Food microbiology 7: 177-198. 